\title{
Neurocognitive Rehabilitation Using Non-Emotionally Charged Material to Re-Learn How to Learn: A Case Report
}

\author{
Murray R. Berkowitz \\ Department of Neuromusculoskeletal Medicine and Osteopathic Manipulative Medicine, Director of Family \\ Medicine/OMM Clerkships, Director of Preventive and Community-Based Medicine, Philadelphia College of \\ Osteopathic Medicine-Georgia Campus, 625 Old Peachtree Road NW, Suwanee, Georgia 30024, USA \\ Email: murraybe@pcom.edu
}

Received December $18^{\text {th }}$, 2013; revised January $16^{\text {th }}$, 2014; accepted February $15^{\text {th }}, 2014$

\begin{abstract}
Copyright (c) 2014 Murray R. Berkowitz. This is an open access article distributed under the Creative Commons Attribution License, which permits unrestricted use, distribution, and reproduction in any medium, provided the original work is properly cited. In accordance of the Creative Commons Attribution License all Copyrights $\mathbb{C}$ 2014 are reserved for SCIRP and the owner of the intellectual property Murray R. Berkowitz. All Copyright (c) 2014 are guarded by law and by SCIRP as a guardian.
\end{abstract}

\begin{abstract}
Cognitive disorders following hypoxic ischemic brain injury involve a variety of disorders including consciousness, behavior, mood and affect, impairment of attention, and memory dysfunction. The case of a 45-year-old former military aviator and engineer, now a physician in residency training, presenting with cognitive difficulties, is described. The patient described having difficulty remembering medical knowledge and feeling fatigued. After almost nine months without any medical intervention and the patient's deteriorating condition, the patient was finally evaluated medically. It was ultimately discovered that the patient suffered from a variety of neurologic impairments that were the direct result of exposures to various toxic substances during his military service. Significant diagnoses included hypoxic ischemic brain damage, severe mixed sleep apnea, and cognitive disorder NOS. Relevant literature about the application of neurocognitive rehabilitation and retraining to treating patients suffering from brain injuries is discussed. The overlap of the neuroscience of emotion with cognitive learning and how emotion and affect impacts learning and education is presented. This case also serves to demonstrate the application of learning and cognition to individual differences and disabilities. Further research is needed to evaluate whether this result is reproducible and generalizable to other patients with similar presenting signs and symptoms.
\end{abstract}

Keywords: Neurocognitive Rehabilitation; Hypoxic Ischemic Brain Damage; Learning; Effects of Emotion on Learning; Re-Learning How to Learn

\section{Introduction}

Bhatoe stated that cognitive disorders following hypoxic ischemic brain injury involve a variety of disorders including consciousness, behavior, mood and affect, impairment of attention, and memory dysfunction (Bhatoe, 2011). There are many etiologies of anoxic and hypoxic brain injuries, consequently no epidemiologic data are known. There are similarities between the sequelae and symptoms demonstrated by patients who sustained traumatic brain injury (TBI) and anoxic/hypoxic brain injury. Relevant literature about the application of neurocognitive rehabilitation and retraining to treating patients suffering from brain injuries is discussed below.

The 1980s saw the development of cognitive rehabilitation. Diamant and Hakkaart integrated various analytical information processing models of cognitive rehabilitation concluding that these models are based on the principles of "functional specificity", "functional hierarchy", and "training circuits (tracks)" (Diamant, 1989). Cullen and associates reported that patients with anoxic brain injury are slower to recover than patients with traumatic brain injury (TBI) (Cullen, 2009). Rajan and associates reported structured, systematic cognitive retraining to be effective in ameliorating cognitive dysfunction in patients suffering from hypoxic brain damage (Rajan, 2010).
Independent studies have demonstrated the effectiveness of functional magnetic resonance imaging (fMRI) in measuring neural changes in cognitive rehabilitation and reported positive association and increased neural connectivity following cognitive rehabilitation (DeGutis, 2007; Minnebusch, 2009; Dalrymple, 2011). Samuel reported that cognitive rehabilitation techniques are more successful with patients suffering from "reversible" brain injuries while it is only marginally successful in patients suffering from "progressive" brain injuries (Samuel, 2008). Fernandez-Ballesteros and associates have studied cognitive plasticity and learning potential in the elderly and have found that there is a positive association of the effects of learning training in normal elderly, and in elderly patients with both mild cognitive impairment (MCI) and Alzheimer's disease (Fernandez-Ballesteros, 2003; Fernandez-Ballesteros, 2012). SalesGalan and associates found measuring cognitive plasticity to be useful in helping identify patients with MCI (Sales-Galan, 2013). Berlucchi discussed that all areas of the brain retain plasticity and even neurogenesis throughout life, even in old age (Berlucchi, 2011).

Dams-O'Connor and Gordon recognized the role of emotion and its impact on cognitive rehabilitation of patients with TBI. "Due to the various cognitive, physical, and emotional changes 
after a TBI, individuals with TBI are constantly confronted with things they cannot do as well as or the same way they did before the accident. Each time this happens, they are confronted with the discrepancy between previous and current abilities, which can cause overwhelming feelings of loss and trigger frustration, depression, and anxiety." They cite studies showing that "emotional regulation appears to be an important mitigating factor in the impact of cognitive rehabilitation, and cognitive interventions that include training in emotional control tend to have a strong impact on functional outcomes" (Dams-O'Connor, 2010).

The case below illustrates how emotional considerations both negatively and positively impact the neurocognitive rehabilitation of a previously extremely highly intelligent and functioning, professionally and socially successful patient. The case will be discussed with emphasis on the application of using positively charged emotional constructs to enhance the patient's ability to re-learn how to learn.

\section{Case}

A 45-year-old former military aviator and engineer, now a physician in residency training, presented with cognitive difficulties. The patient described having difficulty remembering medical knowledge and feeling fatigued. The patient reported to his supervising attending physician these concerns and was summoned before the clinic director who admonished the patient to perform better. After almost nine months without any medical intervention and the patient's deteriorating condition, the patient was finally evaluated medically. It was ultimately discovered that the patient suffered from a variety of neurologic impairments that were the direct result of exposures to various toxic substances during his military service. Significant diagnoses include hypoxic ischemic brain damage, severe mixed sleep apnea, and cognitive disorder NOS. Based on neuropsychological testing, the patient's IQ declined from 186 to 133 . The patient's blood oxygenation saturation declined to between $70 \%$ and $76 \%$ while "asleep"; note that patients with blood oxygenation saturation levels of between $88 \%$ - 90\% are typically placed in the intensive care unit (ICU) and intubated. The patient was placed in a stressful situation to perform duties as a resident physician while struggling to remember medical information. No attempt at neurocognitive rehabilitation was ever performed by the military health care system and the patient received a medical discharge from military service due to his service-connected disabilities.

The patient attempted to "re-learn" his medical knowledge, but the emotional distress of having had a previously excellent military career (including early, "below-the-zone" promotions and the awarding of numerous military awards and decorations) ending by not being able to recall medical information, was determined as interfering with the patient being able to re-learn medical knowledge. The patient was started on a protocol of attempting to "re-learning how to learn" by studying material involving advancing his aviation knowledge. The emotional component of learning aviation had previously been a very positive experience. The patient was able to re-learn both aviation "book knowledge" and "hands-on" performance knowledge and was also able to learn "new" aviation-related knowledge and skills, albeit as a civilian. Following this, the patient was able to apply the techniques he had employed in both "re-learning” older aviation knowledge and skills and learning "new" aviation knowledge and skills to re-learning medical knowledge and information. The patient ultimately earned a Master of Public Health degree and achieved Board Certification in three medical specialties.

\section{Discussion}

Hypoxic ischemic brain injuries often have the unfortunate sequelae of dysfunctions of consciousness, behavior, mood and affect, impairment of attention, and memory. This patient clearly presented with these problems, fully consistent with Bhatoe above. Further, Bhatoe stated that "Recovery ... is to be anticipated over one or two years” (Bhatoe, 2011). This patient is also consistent with the cases presented by Samuel in considering restorative vs. compensatory rehabilitation. Samuel's findings are limited in that they are based upon only two cases -one demonstrating each of the reversible and progressive conditions-and, while illustrated by this case, may not be generalizable (Samuel, 2008).

This patient, in working with previously positively emotional component to re-learn how to learn (i.e., aviation), is fully consistent with Wilson and associates, who reported the positive results of a case of a patient suffering from severe head injury and vascular involvement as a result of a motor vehicle accident using a goal planning approach to cognitive rehabilitation. The Wilson case demonstrated the success of applying a holistic, goal planning approach in patients with non-progressive brain injuries (Wilson, 2002). Levine and associates reported a largescale randomized trial using goal-planning and hierarchies to increase executive deficits. They reported the gains based upon simulated real-life tasks (SRLTs) "were maintained at longterm follow-up” (Levine, 2007). Craik, Winocur, and associates reported improvements in memory encoding and retrieval based on a blocked randomized controlled trial of cognitive training (Craik, 2007; Winocur, 2007). Clearly, this patient regaining his medical knowledge and subsequently earning a Master of Public Health and achieving board certification in three medical specialties is consistent with the long-term gains reported by Craik, Levine, and Winocur above.

McDonald and associates reported the results of a small, pilot cross-over study on the use of external memory aids to improve performance in subjects suffering from impaired memory as a result of acquired brain injuries (McDonald, 2011). This patient only needs minimal use of external memory aids.

This patient is also clearly demonstrates the positive impact of the Dams-O'Connor and Gordon role of emotion and its impact on cognitive rehabilitation of patients with brain injury, albeit with hypoxic ischemic brain injury. The patient in this case clearly confronted with initially not being able to do things he previously was able to do (i.e., recall medical knowledge) and was frustrated every time he tried to re-learn his lost medical knowledge. The negative emotional impact of having lost his career as a military physician triggered the feelings of loss, frustration, and anxiety described by Dams-O'Connor and Gordon. These negative emotions exacerbated the patient's difficulties in achieving a return to function. Immordino-Yang and Damasio describe the overlap of the neuroscience of emotion with cognitive learning and discuss how emotion and affect impacts learning and education. They report research showing that learning, attention, memory, and decision-making "are both profoundly affected by and subsumed within the processes of emotion" (Immordino-Yang, 2007). This patient is again fully 
consistent with these findings. This patient is also fully consistent with the findings of brain plasticity in later life reported by Berlucchi (Berlucchi, 2011).

\section{Summary and Conclusion}

This case shows how positive emotional component of cognition provides a template for re-learning how to learn in patients suffering from hypoxic ischemic brain injury. Had this been done earlier in the course of treatment and cognitive rehabilitation, preferably as soon as practical after the patient had presented with cognitive symptoms, this patient's condition might not have deteriorated to the extent it did and the patient may have returned to function earlier. This case also serves to demonstrate the application of learning and cognition to individual differences and disabilities. Further research is needed to evaluate whether this result is reproducible and generalizable to other patients with similar presenting signs and symptoms.

\section{REFERENCES}

Berlucchi, G. (2011). Brain plasticity and cognitive neurorehabilitation. Neuropsychological Rehabilitation, 21, 553-559. http://dx.doi.org/10.1080/09602011.2011.573255

Bhatoe, H. S. (2011). The hypoxic-ischemic brain injury: Beyond semantics. Indian Journal of Neurotrauma, 8, 65-66. http://dx.doi.org/10.1016/S0973-0508(11)80001-8

Craik, F. I. M., Winocur, G., Palmer, H., Binns, M.A., Edwards, M., Bridges, K., Glazer, P., Chavannes, R., \& Stuss, D. T. (2007). Cognitive rehabilitation in the elderly: Effects on memory. Journal of the International Neuropsychological Society, 13, 132-142. http://dx.doi.org/10.1017/S1355617707070166

Cullen, N. K., Crescini, C., \& Bayley, M. T. (2009). Rehabilitation outcomes after anoxic brain injury: A case-controlled comparison with traumatic brain injury. PM \& R Journal, 1, 1069-1076. http://dx.doi.org/10.1016/j.pmrj.2009.09.013

Dalrymple, K. A., Oruc, I., Duchaine, B., Pancaroglu, R., Fox, C. J., Iaria, G., Handy, T. C., \& Barton, J. J. S. (2011). The anatomic basis of the right face-selective N170 in acquired prosopagnosia: A combined ERP/fMRI study. Neuropsychologia, 49, 2553-2563. http://dx.doi.org/10.1016/j.neuropsychologia.2011.05.003

Dams-O’Connor, K., \& Gordon, W. A. (2010). Role and impact of cognitive rehabilitation. Psychiatric Clinics of North America, 33, 893904. http://dx.doi.org/10.1016/j.psc.2010.08.002

DeGutis, J. M., Bentin, S., Robertson, L. C., \& D’Esposito, M. (2007). Functional plasticity in ventral temporal cortex following cognitive rehabilitationof a congenital prosopagnosic. Journal of Cognitive Neuroscience, 19, 1790-1802. http://dx.doi.org/10.1162/jocn.2007.19.11.1790

Diamant, J. J., \& Hakkaart, P. J. W. (1989). Cognitive reghabilitation in an information-processing perspective. Cognitive Rehabilitation, 22-29.

Fernandez-Ballesteros, R., Botell, J., Zamarron, M. D., Molina, M. A., Cabras, E., Schettini, R., \& Tarraga, L. (2012). Cognitive plasticity in normal and pathological aging. Clinical Interventions in Aging, 7, 15-25. http://dx.doi.org/10.2147/CIA.S27008

Fernandez-Ballesteros, R., Zamarron, M. D., Tarraga, L., Moya, R., \& Iniguez, J. (2003). Cognitive plasticity in healthy, mild cognitive impairment (MCI) subjects and Alzheimer's disease patients: A research project in Spain. European Psychologist, 8, 148-159. http://dx.doi.org/10.1027//1016-9040.8.3.148

Immordino-Yang, M. H., \& Damasio, A. (2007). We feel, therefore we learn: the relevance of affective and social neuroscience to education. Mind, Body, and Education, 1, 3-10. http://dx.doi.org/10.1111/j.1751-228X.2007.00004.x

Levine, B., Stuss, D. T., Winocur, G., Binns, M. A., Fahy, L., Mandic, M., Bridges, K., \& Robertson, I. H. (2007). Cognitive rehabilitation in the elderly: Effects on strategic behavior in relation to goal management. Journal of the International Neuropsychological Society, 13, 143-152. http://dx.doi.org/10.1017/S1355617707070178

McDonald, A., Haslam, C., Yates, P., Gurr, B., Leeder, G., \& Sayers, A. (2011). Google calendar: A new memory aid to compensate for prospective memory deficits following acquired brain injury. Neuropsychological Rehabilitation, 21, 784-804. http://dx.doi.org/10.1080/09602011.2011.598405

Minnebusch, D. A., Suchan, B., Koster, O., \& Daum, I. (2009). A bilateral occipitotemporal network mediates face perception. Behavioural Brain Research, 198, 179-185. http://dx.doi.org/10.1016/j.bbr.2008.10.041

Rajan, J., Udupa, S., \& Bharat, S. (2010). Hypoxia: Can neuropsychological rehabilitation attenuate neuropsychological dysfunction. Indian Journal of Psychological Medicine, 32, 65-68. http://dx.doi.org/10.4103/0253-7176.70544

Sales-Galan, A., Melendez-Moral, J. C., \& Mayordomo-Rodriguez, T. (2013). Using a cognitive plasticity measure to detect mild cognitive impairment. Archives of Clinical Neuropsychology, 1-8.

Samuel, R. (2008). Cognitive rehabilitation for reversible and progresssive brain injury. Indian Journal of Psychiatry, 50, 282-284. http://dx.doi.org/10.4103/0019-5545.44752

Wilson, B. A., Evans, J. J., \& Keohane, C. (2002). Cognitive rehabilitation: A goal-planning approach. Journal of Head Trauma Rehabilitation, 17, 542-555. http://dx.doi.org/10.1097/00001199-200212000-00006

Winocur, G., Craik, F. I. M., Levine, B., Robertson, I. H., Binns, M. A., Alexander, M., Black, S., Dawson, D., Palmer, H., McHugh, T., \& Stuss, D. T. (2007). Cognitive rehabilitation in the elderly: Overview and future directions. Journal of the International Neuropsychological Society, 13, 166-171.

http://dx.doi.org/10.1017/S1355617707070191 